\title{
Evaluation of balance in chronic obstructive pulmonary disease with practical tests
}

\author{
Onur Yazici ${ }^{1}$, Emel Ceylan ${ }^{1}$, Senay Demir Yazici ${ }^{2}$, and Sule Tas Gulen ${ }^{1}$ \\ ${ }^{1}$ Adnan Menderes University School of Medicine \\ ${ }^{2}$ Atatürk State Hospital
}

October 12, 2020

\begin{abstract}
Introduction: Patients with chronic obstructive pulmonary disease (COPD) have impaired balance and hence fall more frequently. Easy application of balance evaluation tests can prevent the loss of time in the outpatient clinic for patients with COPD. Therefore, we compared the effectiveness of the Berg Balance Scale (BBS) test, a relatively more recommended but time-consuming test, with easy-to-perform tests such as the Single Leg Stance (SLS), Five Repetition Sit to Stand (5STS), Four Step Square (FSS), and Timed Up and Go (TUG) tests. Methods: A total of 71 patients with COPD and 70 control subjects with matching demographic characteristics were included in the study. All patients undertook pulmonary function tests and their scores of 6-min walk-test (6MWT), modified Medical Research Council (mMRC) dyspnea scale, COPD assessment questionnaire (CAT), and body mass index (B) were recorded. In addition, the severity of airway obstruction (O), the dyspnea severity (D), and exercise capacity (E) (BODE) index scores were measured. All patients with COPD were classified into 4 groups, A to D, based on the GOLD 2017 criteria. All subjects undertook the BBS, SLS, 5STS, Four Step Square Test, and TUG tests. Results: Balance tests revealed increased balance impairment in patients with COPD in comparison with the control subjects $(\mathrm{P}<0.05)$. The BBS, SLS, Four Step Square Test $(\mathrm{FSST})$, and TUG examinations suggested that balance impairment was frequent in Groups $\mathrm{C}$ and D patients with COPD $(\mathrm{P}<0.05)$. TUG and FSST had the highest correlation with BBS ( $r=-0.812$ and -0.842 and $\mathrm{P}<0.001$ and $<0.001$, respectively). The results of TUG and FSST were the closest to those of BBS test. Conclusions: TUG and FSST tests can be applied for the assessment of bodily balance status among patients with COPD in outpatient clinics.
\end{abstract}

\section{INTRODUCTION}

Owing to its systemic effects, several chronic obstructive pulmonary disease (COPD) are linked with many comorbidities. Although comorbidities such as cachexia, skeletal muscle weakness, and osteoporosis are well-known, the balance impairment and falls resultant from these issues are not adequately emphasized. However, the frequency of the loss of balance and falls is more among patients with COPD than in the normal population. ${ }^{1-3}$ Several factors are known to contribute to balance impairment in patients with COPD. Of which, the most remarkable factor is developing muscle atrophies due to decreased daily life activities. Quadriceps muscle weakness due to immobilization in patients with COPD is a major risk factor for balance impairment and fall. Hypoxia, oxidative stress, and systemic inflammation play major roles in the development of muscle atrophy. ${ }^{4,5}$ Fractures due to impaired balance and falls increase the resultant morbidity and mortality as a result of decreased quality of life, bed confinement, increased muscle atrophy, and complications such as thromboembolism. Therefore, it has been recently emphasized that the balance impairment should be treated as a part of pulmonary rehabilitation treatment plan. ${ }^{6}$

Several tests are available for the evaluation and assessment of balance impairment and fall risk in patients with COPD. Although the Berg Balance Scale (BBS) and Balance Evaluation System (BES) tests are the most recommended tests of all, ${ }^{6}$ these tests are very complicated and time consuming as they evaluate 
numerous factors that affect the bodily balance of a patient. As a result, it is difficult to apply these tests to the routine outpatient evaluation of patients with COPD. Identifying patients at a high risk of balance impairment and fall with the application of easy-to-use tests at the beginning, followed by performing more detailed tests later is expected to save the times of both the patient and the healthcare worker. The initial tests should be easy to understand and practical. Single Leg Stance (SLS) test, Five Repetition Sit to Stand Test (5STS), Four Step Square Test (FSST), and Timed Up and Go (TUG) test are some easy-to-use tests that can effectively evaluate balance impairment and fall risk.

In this study, we evaluated balance impairment in patients with COPD using tests that are easier to perform than the BBS test.

\section{METHODS}

\subsection{Study design and patients}

In this study, a total of 71 patients with COPD admitted to the Adnan Menderes University outpatient clinics of chest diseases during March 2018-2019 and diagnosed with COPD with reference to the Global Initiative for Chronic Obstructive Lung Disease (GOLD) guidelines ${ }^{7}$ were included in the study. The control group contained 70 individuals aged [?]40 years with normal respiratory function test results. Subjects with communication problems, postural orthostatic hypertension, unstable cardiac disease, hearing or visual impairments, any skeletal or neurological problems that may cause falls, as well as those on sedatives (such as antidepressants), those operated from the lower limbs, those with balance and gait impairments, and those using assistive walking devices were excluded from the study. The study was approved by the Adnan Menderes University Non-invasive Clinical Studies Ethics Committee (No: 2018/1297), and the study was conducted in accordance with the principles of the Declaration of Helsinki. All participants provided their informed signed consent forms.

\subsection{Collection of data}

Data on the age, gender, body mass index (BMI), smoking status, and fall history in the last 1 year were recorded for all subjects. The respiratory function tests were performed with reference to the American Thoracic Society/European Respiratory Society criteria, and the parameters forced vital capacity (FVC), forced expiratory volume first second (FEV1), and the ratio of FEV1/FVC were recorded. ${ }^{8}$ In addition, the scores of 6-min-walk-test (6MWT), modified Medical Research Council (mMRC) dyspnea scale, COPD assessment questionnaire (CAT), BMI (B), the severity of airway obstruction (O), the dyspnea severity (D), and exercise capacity (E) (BODE) index were also collected. All patients with COPD were classified into 4 groups based on the GOLD 2017 criteria. Accordingly, the patients with high frequency of symptoms were assigned to Group B or D, while those with high risk of exacerbation were assigned to Group C or D. ${ }^{9}$ All subjects were informed about the balance tests, and based on how the tests were performed, BBS, SLS, 5STS, FSST, and TUG tests were performed in the physical therapy and rehabilitation outpatient clinic.

\subsection{Evaluation of balance impairment}

\subsubsection{Single Leg Stance (SLS) Test}

This test was used to assess the static balance. Briefly, the subject was asked to stand on 1 leg, with the other leg flexed to 90 degrees. If the raised foot touched the standing leg or the ground or if the subject jumped or hopped on the standing leg or held on to something, the test was terminated and the time was recorded. The subjects' performed this test twice, once with eyes open and once closed. The test was repeated thrice and the best time was recorded. Values $<30 \mathrm{~s}$ with open eyes indicated impaired balance. ${ }^{10,11}$

\subsubsection{Five Repetition Sit to Stand Test}

This test was used to evaluate the balance and muscle strengths of the lower limbs. Briefly, the subject was made to sit on a chair of an average height $43 \mathrm{~cm}$ (17 in) with his/her back rested, arms crossed on the chest, and feet flat on the ground. When prompted, the subject got up and sat 5 times in this position as 
fast as possible. The total time taken to complete this sequence determined the test result. The results $>13$ s indicated impaired balance. ${ }^{12}$

\subsubsection{Four Step Square (FSS) Test}

This test was used to evaluate the dynamic balance and the ability to change direction while walking stepby-step. Four squares were placed on a flat surface, and the squares were numbered from 1 to 4 . The subject stood in the $1^{\text {st }}$ square with both his/her feet flat on the ground. The subject was then asked to walk in the squares to square number $1,2,4$, and 3 in a clockwise direction and then returning back and beginning again from the square number 3 to 4,2 , and 1 . The subject was next asked to move as fast as he/she could, without touching the outer lines and stepping in the squares with both the feet. The test was repeated twice, and the best time was recorded. Scores $>15 \mathrm{~s}$ indicated impaired balance and the risk of fall. ${ }^{11,13-15}$

\subsubsection{Timed Up and Go Test}

This test was used to assess the fall risk and mobility. The subject was asked to sit on a chair and then stand up, walk 3-m (10 ft) ahead to a marked area, and then return and sit on the chair again. The total time taken to complete this sequence was the test result. The test was repeated once more and the outcome was recorded. The threshold for the subjects' high risk of fall was $13.5 \mathrm{~s}$. Time $>13.5 \mathrm{~s}$ was considered to indicate a high risk of falling. ${ }^{16}$

\subsubsection{Berg Balance Scale Test}

This is the most commonly used test and also the gold standard for evaluating static and dynamic balances in adults. ${ }^{17,} 18$ This test involves 14 tasks, and the subject's performance was observed during the sequence of sitting down, standing up, performing different types of standing action on feet (such as with eyes open and closed, at tandem, on 1 leg, etc.), then transferring, reaching forward and stretching, and finally turning around. The performance was graded on a scale of $0-4$. The total score was $0-56$. Higher scores reflected better balance. The threshold was 46 , and scores $<46$ indicated a risk of falling. ${ }^{19}$

\subsection{Statistical Analysis}

The data were analyzed by using the SPSS package program (SPSS 23, IBM Corp. Released 2015. IBM SPSS Statistics for Windows, Version 23.0. Armonk, NY, USA). The suitability of variables to normal distribution was determined using the Kolmogorov-Smirnov test. Normally distributed continuous variables were expressed as mean \pm standard deviation, while those without normal distribution were expressed as median (25\%-75\% percentile). Categorical variables were expressed in numbers and percent. Student t-test was performed for normally distributed continuous variables, while Mann-Whitney U-test was performed for those without normal distribution. Pearson's chi-square and Fisher's exact tests were used to evaluate categorical variables. For correlation analysis, the Pearson correlation coefficient was used when the preconditions of the parametric test were met, and the Spearman correlation coefficient was used when the preconditions were not met. A $\mathrm{P}$ value $<0.005$ was considered to reflect statistical significance.

\section{RESULTS}

A total of 71 patients with COPD of mean age $64.73 \pm 8.16$ years and 70 control subjects of mean age 63.07 \pm 7.42 years were included in this study. The subjects included $91.5 \%(\mathrm{n}=65)$ men in the COPD group and $92.9 \%(\mathrm{n}=65)$ men in the control group $(P=0.772)$. The BMI of the subjects in both the groups were similar $(P=0.114)$. However, $26.8 \%(\mathrm{n}=19)$ of the patients with COPD were active smokers, while the corresponding rate was $12.9 \%(\mathrm{n}=9)$ in the control group. The COPD group patients smoked more than the control group subjects $(P=0.004)$. The COPD patients had lower 6 -MWT scores and their pulmonary function test parameters were worse, but their mMRC, CAT, and BODE index scores were higher than those of the control subjects $(P<0.001)$. In the COPD group, $24(43.8 \%)$ patients had a fall history in the past year, while $10(24.3 \%)$ subjects in the control group had a fall history $(P=0.007)$. The patient characteristics are illustrated in Table 1. 
Based on the balance tests, SLS revealed an balance impairment in $57(80.3 \%)$ of the patients in the COPD group and in $35(50 \%)$ of the subjects in the control group $(P<0.001)$. Significantly shorter times for standing on $1 \mathrm{leg}$ were recorded for the COPD group than for the control group $(P<0.001)$. 5STS test results revealed that $66(93 \%)$ of the patients with COPD and $35(50 \%)$ of the control subjects had balance impairment $(P<0.001)$. The time for finishing the test was significantly longer for the patients with COPD than for the control subjects $(P<0.001)$. In FSST, balance impairment was detected in $49(69 \%)$ patients with COPD and in 30 control subjects $(42.9 \%)(P=0.002)$. The time for finishing the test was significantly longer in the COPD group as compared to that in the control group $(P=0.001)$. In TUG test, balance impairment was detected in $38(53.5 \%)$ and $23(32.9 \%)$ of the patients with COPD and control subjects, respectively $(P=0.013)$. The time for the test was significantly longer in the COPD group $(\mathrm{P}=0.007)$. The BBS test revealed that $32(45.1 \%)$ and $19(27.1 \%)$ of the patients with COPD and control subjects, respectively, suffered from balance problems $(P=0.027)$. The BBS scores were significantly lower in the COPD group $(P=0.003)$. The test results for COPD and control groups are depicted in Table 2 .

Based on the weight of the participants, the SLS, FSST, TUG, and BBS tests detected more balance impairment in the Group C and D patients with COPD. However, no significant difference was noted in terms of the 5STS results (Table 3). The median values of the 5STS results of Group C and D patients with COPD were significantly longer than those of Group A and B patients $(P<0.001)$. Group $\mathrm{C}$ and D patients with COPD had more cases of falling in the last 1 year in comparison to Group A and B patients $(P<0.001)$ (Table 3).

BSS positively correlated with SLS but negatively correlated with 5STS, FSST, and TUG, however, FSST and TUG had the highest correlation with BBS ( $\mathrm{r}=-0.812$ and -0.842 and $\mathrm{P}<0.001$ and $<0.001$, respectively) (Figure 1).

BBS and SLS results showed a negative correlation with age, CAT, mMRC, and BODE scores, but a positive correlation with FVC (L and \%), FEV1 (L and \%), FEV1/FVC, and 6-MWT results. The 5STS, FSST, and TUG test results showed a positive correlation with age, CAT, mMRC, and BODE scores, while correlation between the results of FVC (L and \%), FEV1 (L and \%), FEV1/FVC, and 6-MWT and the results of 5STS, FSST, and TUG results was negative (Table 4).

\section{DISCUSSION}

Although balance impairment in patients with COPD seems to be less important than the other lifethreatening symptoms of this disease, the fall and fractures resulting from the balance impairment may lead to a decrease in the quality of life and an increase in morbidity and mortality. Recent studies have shown that balance impairment and falls are frequent in patients with COPD. ${ }^{1,20}$ Numerous tests ranging from simple clinical tests to more complex tests conducted using diverse devices in the laboratory environment have been utilized to evaluate the balance issues in patients. The decision regarding which test should be used has changed with time, conditions, and personal experiences. ${ }^{21-23}$ Studies on balance have generally been performed among the elderly, post-operative patients, post-stroke term, and rheumatic disease patients. However, the number of studies investigating balance issues in patients with COPD is low, and even these studies have investigated a small cohort of patients and utilized only a small portion of balance assessment tests. In pulmonary diseases outpatient clinic, it would be more appropriate for the physicians to select easy-to-perform balance assessment tests to enable focusing on the underlying primary disease for the treatment of patients with COPD who are considered to have balance impairment should be evaluated with the shorter tests at first presentation, and these patients should be directed and evaluated in pulmonary rehabilitation. Hence, patients should be evaluated with more complicated tests to reveal the problem in balance mechanism in order to plan and implement a rehabilitation program accordingly.

BBS is the gold standard in balance assessment and also one of the most frequently applied tests for assessing balance in patients with COPD. Recent studies have shown that patients with COPD have significantly lower BBS scores than the normal population. ${ }^{1,5}$ In our study, we found that $32(45.1 \%)$ of the patients with COPD had balance impairment as per the BBS test. However, since it takes approximately 15 min to perform the 
BBS test, it is difficult to perform it daily in the complex environment of an outpatient clinic. ${ }^{6}$

The past studies assert that SLS test is one of the most frequently employed tests for the assessment of balance impairment in patients with COPD, with more balance impairment detected by SLS in patients with COPD relative to that in control subjects. ${ }^{24-26}$ In our study, SLS test could detect balance impairment in $57(80.3 \%)$ of the patients with COPD. However, when compared to the BBS test, SLS test could detect balance impairment in more number of patients, indicating that SLS is less selective than BBS.

In our study, we detected balance impairment in 66 (92.9\%) of the patients with COPD by using the 5STS test. The comparative rate was $50 \%$ in the control group. This difference may be attributed to the fatigue of weakened quadriceps muscles in patients with COPD after 5 times of "sitting down and standing up" sequence that extends the times required to finish the test. Since this test has low selectivity with respect to assessing balance impairment in patients with COPD, we suggest that other tests should be performed before STS to eliminate non-essential steps.

We detected balance impairment in $49(69 \%)$ of our patients by using the FSST test. The reason for the greater frequency of detection relative to that by BBS can be the difficulty in comprehending the FSST test. However, the results of FSST are more consistent with BBS in terms of detecting balance impairment when compared to SLS and 5STS. FSST had the second highest correlation with BBS. In addition, while BBS requires $15 \mathrm{~min}$ for completion, FSST can be completed in $5 \mathrm{~min}$, which favors the preference for the latter.

In our study, balance impairment was detected in 38 (53.5\%) of the patients with TUG test. Among all the tests, TUG gave the closest results with BBS in terms of detecting balance impairment. Morover TUG had the highest correlation with BBS. This test evaluates patients in 3 stages. The test first evaluates the quadriceps muscles when the patient stands up from the chair and sits; this muscle is the most-affected peripheral muscle group in patients with COPD. In the next 2 stages, the balance impairment observed during the daily life activities is evaluated by walking the patient for $3-\mathrm{m}(10 \mathrm{ft})$ and back. Thus, the static and dynamic balances are evaluated by this test. Hence, we believe that this test can produce more objective results in patients with COPD in comparison with other easy-to-perform tests.

SLS and 5STS can evaluate static balance, while TUG and FSST can evaluate both static and dynamic balances. We believe that it would be more accurate to evaluate dynamic balance together with static balance when evaluating the balance issue in patients with COPD, since evaluation of only the static balance will bypass maneuvers that maintain balance during a regular gait. In our study, the closest results to BBS were obtained with TUG and FSST.

In our study, balance impairment and fall history in Group C and D patients with COPD were more frequent than those in Group A and B. COPD exacerbations negatively affect the muscle functions and physical activity levels. As a result, the incidence of fall increased. ${ }^{27}$ Since Groups C and D had higher frequency of exacerbation, these groups showed more balance impairment and an increased incidence of falls in both the groups.

The risk of balance impairment and falls in patients with COPD are not considered very important by healthcare professionals and patients. However, in our study, we observed that balance impairment and frequency of falls increased in patients with COPD and that the balance test scores could be correlated with functional parameters such as patient's pulmonary function test parameters, CAT, mMRC, and BODE scores. In this case, we speculate that evaluating patients with COPD with balance tests is a useful approach toward determining balance impairment and risk of falls and for predicting the functional status.

The main limitation of our study was that we did not use the BES and Activities Specific Balance Confidence Scale with BBS in the comparison assessment with SLS, 5STS, FSST, and TUG tests. The former tests are more detailed for the evaluation of balance. However, since all of these 3 tests are relatively more time consuming to apply simultaneously, BBS, which is the most frequently used test, was preferred in this study.

\section{CONCLUSIONS}


Easy-to-apply balance tests can be effective for patients with COPD who are at risk as these tests can help identify body balance issues at the early stages, making rehabilitation initiation possible in a timely manner. Since TUG and FSST tests produced the closest results to BBS test, we believe that they can be used preferably in the outpatient clinic.

\section{DISCLOSURE}

The authors declare no conflict of interest related to this publication

\section{REFERENCES}

1. Beauchamp MK, Sibley KM, Lakhani B, et al. Impairments in systems underlying control of balance in COPD. Chest. 2012;141:1496-1503.

2. Smith MD, Chang AT, Seale HE, Walsh JR, Hodges PW. Balance is impaired in people with chronic obstructive pulmonary disease. Gait Post. 2010;31:456-460.

3. Roig M, Eng J, MacIntyre D, Road JD, Reid WD. Postural control is impaired in people with COPD: an observational study. Physiotherapy Canada. 2011;63:423-431.

4. Barnes PJ, Celli BR. Systemic manifestations and comorbidities of COPD. Eur Respir J. 2009;33(5):11651185 .

5. Tudorache E, Oancea C, Avram C, Fira-Mladinescu O, Petrescu L, Timar B. Balance impairment and systemic inflammation in chronic obstructive pulmonary disease. Int J Chron Obstruct Pulmon Dis. 2015;10:1847-1852.

6. Beauchamp MK. Balance assessment in people with COPD: An evidence-based guide. Chron Respir Dis. 2019;16:1479973118820311.

7. Global Strategy for the Diagnosis, Management and Prevention of COPD, Global Initiative for Chronic Obstructive Lung Disease (GOLD). 2011 revision. Available from: http://www.goldcopd.org (accessed September 1, 2015).

8.Miller MR, Crapo R, Hankinson J, et al. General considerations for lung function testing. Eur Respir J. 2005;26:153-161.

9. Global Strategy for the Diagnosis, Management and Prevention of COPD, Global Initiative for Chronic Obstructive Lung Disease (GOLD) 2017 [c2017 Available from: http://goldcopd.org.

10. Michikawa T, Nishiwaki Y, Takebayashi T, Toyama Y. One-leg standing test for elderly populations. J Orthop Sci. 2009;14(5):675-685.

11. Isik EI, Altug F, Cavlak U. Reliability and validity of four square step test in older adults. Turk J Geriatr. 2015;18(2):151-155.

12. Whitney SL, Wrisley DM, Marchetti GF, Gee MA, Redfern MS, Furman JM. Clinical measurement of sit-to-stand performance in people with balance disorders: validity of data for the Five-Times-Sit-to-Stand Test. Phys Ther. 2005;85(10):1034-1045.

13.Duncan PR, Earhart GM. Four Square Step Test performance in people with Parkinson Disease. J Neurol Phys Ther. 2013;37(1):2-8.

14.Günendi Z, Demirsoy N. Clinical and Computerized Stabilometrical Evaluation of Postural Balance in Postmenopausal Women with Osteoporosis. Turk J Phys Med Rehab. 2007;53:130-133.

15. Dite W, Temple VA. A clinical test of stepping and change of direction to identify multiple falling older adults. Arch Phys Med Rehabil. 2002;83(11):1566-1571. 
16. Barry E, Galvin R, Keogh C, Horgan F, Fahey T. Is the Timed Up and Go test a useful predictor of risk of falls in community dwelling older adults: a systematic review and meta- analysis. BMC Geriatrics. $2014 ; 14: 14$.

17. Langley FA, Mackintosh SFH. Functional balance assessment of older community dwelling adults: a systematic review of the literature. The Internet Journal of Allied Health Sciences and Practice. 2007;5(4):111.

18. Tyson SF, Connell LA. How to measure balance in clinical practice. A systematic review of the psychometrics and clinical utility of measures of balance activity for neurological conditions. Clin Rehabil. 2009;23(9):824-840.

19. Beauchamp MK, Janaudis-Ferreira T, Parreira V, et al. A randomized controlled trial of balance training during pulmonary rehabilitation for individuals with COPD. Chest. 2013;144(6):1803-1810.

20. Roig M, Eng JJ, MacIntyre DL, et al. Falls in people with chronic obstructive pulmonary disease: an observational cohort study. Respir Med. 2011;105(3):461-469.

21. Balaban Ö, Nacır B, Erdem HR, Karagöz A. The evaluation of balance function. FTR Bil Der. 2009;12:133-139.

22. Herman T, Giladi N, Hausdorff JM. Properties of the 'timed up and go' test: more than meets the eye. Gerontology. 2011;57:203-210.

23. Berg K, Wood-Dauphinee S, Williams JI, Maki B, Gayton D. Measuring Balance in the Elderly - Validation of an Instrument. J Am Geriatr Soc. 1991;39:A65-A.

24. Oliveira CC, Lee A, Granger CL, Miller KJ, Irving LB, Denehy L. Postural control and fear of falling assessment in people with chronic obstructive pulmonary disease: a systematic review of instruments, international classification of functioning, disability and health linkage, and measurement properties. Arch Phys Med Rehabil. 2013;94:1784-1799, e7.

25. Crişan AF, Oancea C, Timar B, FiraMladinescu O, Tudorache V. Balance Impairment in Patients with COPD. PLoS ONE. 2015;10(3):e0120573.

26. Voica AS, Oancea C, Tudorache E, et al. Chronic obstructive pulmonary disease phenotypes and balance impairment. Int J Chron Obstruct Pulmon Dis. 2016;11:919-1925.

27. Spruit MA, Gosselink R, Troosters T, et al. Muscle force during an acute exacerbation in hospitalised patients with COPD and its relationship with CXCL8 and IGFI. Thorax. 2003;58: 752-756.

Table 1. Demographic data of the patients with COPD and control subjects

\begin{tabular}{llll}
\hline Variables & COPD $\mathbf{~}=\mathbf{7 1}$ & Control $\mathbf{~}=\mathbf{7 0}$ & $\mathbf{P}$ \\
\hline Age (years) & $64.73 \pm 8.16$ & $63.07 \pm 7.42$ & 0.208 \\
Gender n (\%) Female & $6(\% 8.5) 65(\% 91.5)$ & $5(\% 7.1) 65(\% 92.9)$ & 0.772 \\
Male & $24.84 \pm 4.24$ & $25.73 \pm 2.07$ & \\
BMI & $(\% 5.6) 48(\% 67.6) 19$ & $33(\% 47.1) 28(\% 40) 9$ & 0.114 \\
Smoking history n (\%) & $(\% 12.9)$ & \\
Never smoker Ex-smoker & $(\% 26.8)$ & & \\
Active smoker & $40(26.25-55)$ & $30(20-40)$ & 0.001 \\
Smoking (packs/year) & $335(227-408.6)$ & $457(408-499)$ & $<0.001$ \\
6MWT (m) & $60(42-73.89)$ & $89.84(84.34-93.77)$ & $<0.001$ \\
6MWT (\%) & $12(8-20)$ & $1(0-2)$ & $<0.001$ \\
CAT & $2(1-2)$ & $0(0-0)$ & $<0.001$ \\
mMRC & $3(1-5)$ & $0(0-0)$ & $<0.001$ \\
BODE & & &
\end{tabular}




\begin{tabular}{llll}
\hline Variables & COPD $\mathbf{n}=\mathbf{7 1}$ & Control $\mathbf{n}=\mathbf{7 0}$ & $\mathbf{P}$ \\
\hline FVC (L) & $2.77 \pm 0.73$ & $3.79 \pm 0.65$ & $<0.001$ \\
FVC (\% pred) & $76.65 \pm 16.07$ & $100.54 \pm 11.79$ & $<0.001$ \\
FEV1 (L) & $1.54(1.12-1.93)$ & $3.11(2.64-3.34)$ & $<0.001$ \\
FEV1 (\%) & $54.27 \pm 15.55$ & $101.78 \pm 12.2$ & $<0.001$ \\
FEV1 FVC & $57(49-64)$ & $78.86(76.62-82.80)$ & $<0.001$ \\
Fall history, n (\%) & $24(33.8)$ & $10(14.3)$ & 0.007 \\
\hline
\end{tabular}

Values are $\mathrm{n}(\%)$, mean \pm standard deviation, or median (25th and 75th interquartile range). Abbreviations: BMI, Body mass index; 6MWT, 6-min walk-test; CAT, COPD assessment test; mMRC, Modified Medical British Research Council dyspnea questionnaire; BODE, BMI (B), airflow obstruction (O), dyspnea (D) and exercise capacity (E); FVC, forced vital capacity; \% pred., percent predicted; FEV1, forced expiratory volume in $1 \mathrm{~s}$.

Table 2. Balance test results of the patients with COPD and control subjects

\begin{tabular}{|c|c|c|c|}
\hline Variables & $\operatorname{COPD} n=71$ & Control $n=70$ & $\mathbf{P}$ \\
\hline $\begin{array}{l}\text { BBS, n (\%) Impaired } \\
\text { balance }(+) \text { Impaired } \\
\text { balance }(-)\end{array}$ & $32(45.1) 39(54.9)$ & $19(27.1) 51(72.9)$ & 0.027 \\
\hline BBS & $48(39-51)$ & $49.5(45-52)$ & 0.003 \\
\hline $\begin{array}{l}\text { SLS, n (\%) Impaired } \\
\text { balance (+) Impaired } \\
\text { balance (-) }\end{array}$ & $57(80.3) 14(19.7)$ & $35(50) 35(50)$ & $<0.001$ \\
\hline SLS (seconds) & $14.3(4.3-25.3)$ & $29.4(20.5-35.1)$ & $<0.001$ \\
\hline $\begin{array}{l}\text { 5STS, n (\%) Impaired } \\
\text { balance (+) Impaired } \\
\text { balance (-) }\end{array}$ & $66(93) 5(7)$ & 3535 & $<0.001$ \\
\hline 5STS (seconds) & $18.2(15.2-22.1)$ & $13.2(12.5-14.4)$ & $<0.001$ \\
\hline $\begin{array}{l}\text { FSST, n (\%) Impaired } \\
\text { balance }(+) \text { Impaired } \\
\text { balance }(-)\end{array}$ & $49(69) 22(31)$ & $30(42.9) 40(57.1)$ & 0.002 \\
\hline FSST (seconds) & $16.3(14.2-20.2)$ & $14.7(13.4-16.3)$ & 0.001 \\
\hline $\begin{array}{l}\text { TUG, n (\%) Impaired } \\
\text { balance (+) Impaired } \\
\text { balance }(-)\end{array}$ & $38(53.5) 33(46.5)$ & $23(32.9) 47(67.1)$ & 0.013 \\
\hline TUG (seconds) & $13.7(12.5-16.5)$ & $13.1(12.2-14.5)$ & 0.007 \\
\hline
\end{tabular}

Values are $\mathrm{n}(\%)$ or median (25th and 75th interquartile range). Abbreviations: BBS, Berg Balance Scale; SLS, Single Leg Stance; 5STS, Five Repetition Sit to Stand Test; FSST, Four Step Square Test; TUG, Timed Up and Go Test.

Table 3. Status of impaired balance and falling in the COPD groups

\begin{tabular}{|c|c|c|c|}
\hline Variables & $\begin{array}{l}\text { COPD Group A and } \\
\text { B } \mathrm{n}=44\end{array}$ & $\begin{array}{l}\text { COPD Group C and } \\
D \mathrm{n}=27\end{array}$ & $\mathbf{P}$ \\
\hline $\begin{array}{l}\text { BBS, n (\%) Impaired } \\
\text { balance (+) Impaired } \\
\text { balance (-) }\end{array}$ & $10(22.7) 34(77.3)$ & $22(81.5) 5(18.5)$ & $<0.001$ \\
\hline
\end{tabular}




\begin{tabular}{|c|c|c|c|}
\hline Variables & $\begin{array}{l}\text { COPD Group A and } \\
\text { B } n=44\end{array}$ & $\begin{array}{l}\text { COPD Group C and } \\
D_{n}=27\end{array}$ & $\mathbf{P}$ \\
\hline BBS & $50(47-52)$ & $48(43-50)$ & $<0.001$ \\
\hline $\begin{array}{l}\text { SLS, n (\%) Impaired } \\
\text { balance (+) Impaired } \\
\text { balance (-) }\end{array}$ & $32(72.7) 12(27.3)$ & $25(92.6) 2(7.4)$ & 0.041 \\
\hline SLS (seconds) & $22.2(12-31.8)$ & $16.3(10.1-25.8)$ & $<0.001$ \\
\hline $\begin{array}{l}\text { 5STS, n (\%) Impaired } \\
\text { balance (+) Impaired } \\
\text { balance (-) }\end{array}$ & $39(88.6) 5(11.4)$ & $27(100) 0(0)$ & 0.149 \\
\hline 5STS (seconds) & $16.2(14.5-19.1)$ & $17(15.2-20.8)$ & $<0.001$ \\
\hline $\begin{array}{l}\text { FSST, n (\%) Impaired } \\
\text { balance }(+) \text { Impaired } \\
\text { balance }(-)\end{array}$ & $26(59.1) 18(40.9)$ & $23(85.2) 4(14.8)$ & 0.021 \\
\hline FSST (seconds) & $15.8(12.8-17.1)$ & $16(13.6-19)$ & $<0.001$ \\
\hline $\begin{array}{l}\text { TUG (n, \%) Impaired } \\
\text { balance (+) Impaired } \\
\text { balance (-) }\end{array}$ & $14(31.8) 30(68.2)$ & $24(88.9) 3(11.1)$ & $<0.001$ \\
\hline TUG (seconds) & $13.1(12-14.8)$ & $13.2(11.7-16.1)$ & $<0.001$ \\
\hline Fall history, n (\%) & $6(13.6)$ & $18(66.7)$ & $<0.001$ \\
\hline
\end{tabular}

Values are n (\%) or median (25th and 75th interquartile range). Abbreviations: BBS, Berg Balance Scale; SLS, Single Leg Stance; 5STS, Five Repetition Sit to Stand Test; FSST, Four Step Square Test; TUG, Timed Up and Go Test; COPD, Chronic Obstructive Lung Disease.

Table 4. Correlation analysis between impaired balance and functional parameters

\begin{tabular}{lllllllllll}
\hline & BBS & BBS & SLS & SLS & TUG & TUG & 5STS & 5STS & FSST & FSST \\
\hline & $\mathbf{r}$ & P & r & P & r & P & r & P & r & P \\
Age & -0.402 & $<0.001$ & -0.580 & $<0.001$ & 0.373 & $<0.001$ & 0.349 & $<0.001$ & 0.513 & $<0.001$ \\
FVC (L) & 0.468 & $<0.001$ & 0.450 & $<0.001$ & -0.417 & $<0.001$ & -0.582 & $<0.001$ & -0.408 & $<0.001$ \\
FVC (\%) & 0.398 & $<0.001$ & -0.329 & $<0.001$ & -0.361 & $<0.001$ & -0.570 & $<0.001$ & -0.336 & $<0.001$ \\
FEV1 (L) & 0.459 & $<0.001$ & 0.507 & $<0.001$ & -0.432 & $<0.001$ & -0.674 & $<0.001$ & -0.426 & $<0.001$ \\
FEV1 (\%) & 0.368 & $<0.001$ & 0.393 & $<0.001$ & -0.360 & $<0.001$ & -0.627 & $<0.001$ & -0.344 & $<0.001$ \\
FEV1/FVC & 0.346 & $<0.001$ & 0.427 & $<0.001$ & -0.349 & $<0.001$ & -0.651 & $<0.001$ & -0.363 & $<0.001$ \\
6MWT(m) & 0.415 & $<0.001$ & 0.385 & $<0.001$ & -0.363 & $<0.001$ & -0.574 & $<0.001$ & -0.394 & $<0.001$ \\
CAT & -0.404 & $<0.001$ & -0.411 & $<0.001$ & 0.372 & $<0.001$ & 0.598 & $<0.001$ & 0.371 & $<0.001$ \\
mMRC & -0.435 & $<0.001$ & -0.417 & $<0.001$ & 0.375 & $<0.001$ & 0.630 & $<0.001$ & 0.379 & $<0.001$ \\
BODE & -0.549 & $<0.001$ & -0.470 & $<0.001$ & 0.485 & $<0.001$ & 0.689 & $<0.001$ & 0.452 & $<0.001$ \\
\hline
\end{tabular}

Abbreviations: FVC, forced vital capacity; \% pred., percent predicted; FEV1, forced expiratory volume in 1 s; 6MWT, 6-min walk-test; CAT, COPD assessment test; Mmrc, Modified Medical British Research Council dyspnea questionnaire; BODE, BMI (B), airflow obstruction (O), dyspnea (D) and exercise capacity (E); BBS, Berg Balance Scale; SLS, Single Leg Stance; TUG, Timed Up and Go Test; 5STS, Five Repetition Sit to Stand Test; FSST, Four Step Square Test. 

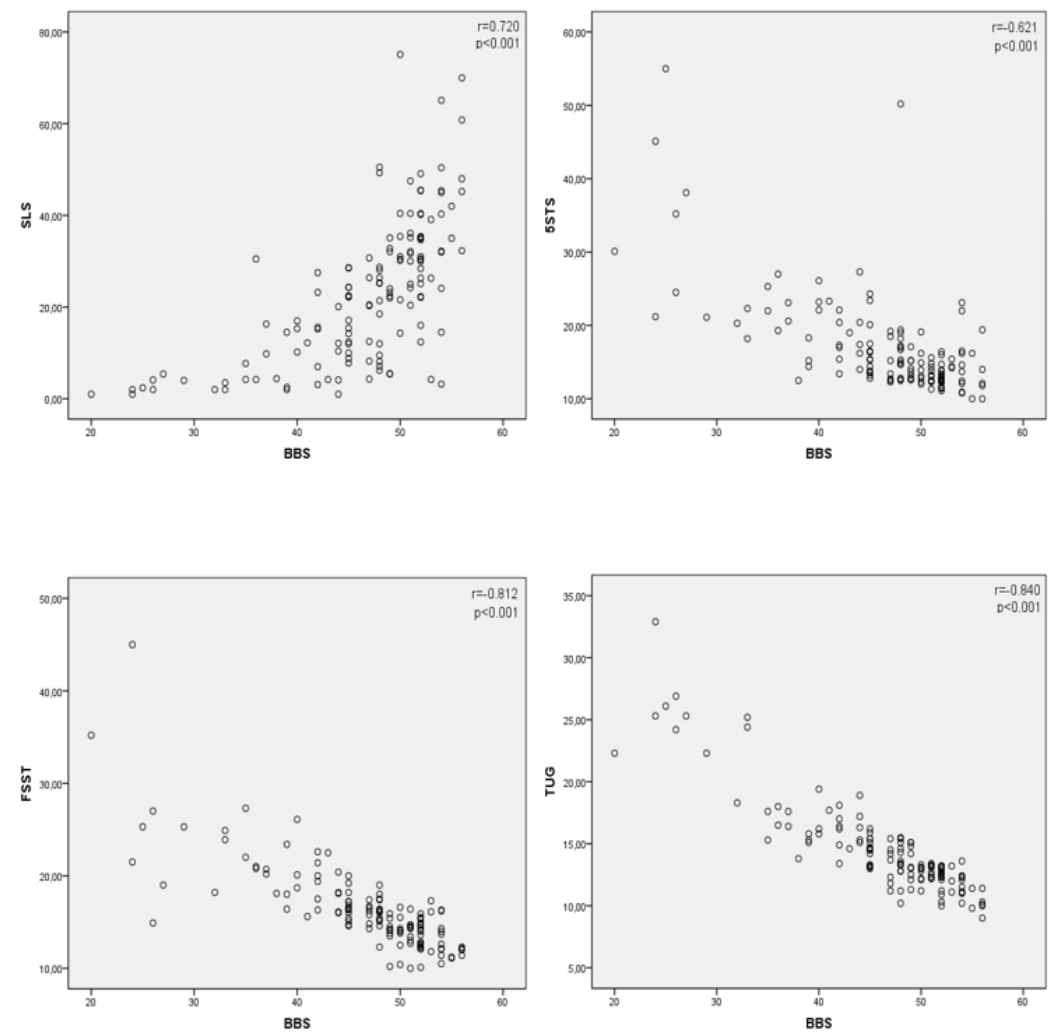

FİGURE 1 . Correlation between BBS results with SLS, 5STS, FSST and TUG results

Abbreviations: BBS, Berg Balance Scale; SLS, Single Leg Stance; 5STS, Five Repetition Sit to Stand Test; FSST, Four Step Square Test, TUG, Timed Up and Go Test

\section{Hosted file}

Figure.pdf available at https://authorea.com/users/366262/articles/486040-evaluation-ofbalance-in-chronic-obstructive-pulmonary-disease-with-practical-tests

\section{Hosted file}

Tables.pdf available at https://authorea.com/users/366262/articles/486040-evaluation-ofbalance-in-chronic-obstructive-pulmonary-disease-with-practical-tests 